13

\title{
Моделирование формирования сжатого состояния электронного пучка в замкнутой трубе дрейфа при токе пучка, меньшем предельного
}

\author{
(C) А.Е. Дубинов, ${ }^{1,2}$ В.П. Тараканов ${ }^{3,4}$ \\ ${ }^{1}$ Российский федеральный ядерный центр - Всероссийский научно-исследовательский \\ институт экспериментальной физики, \\ 607188 Саров, Нижегородская обл., Россия \\ ${ }^{2}$ Саровский фризико-технический институт, \\ 607189 Саров, Нижегородская обл., Россия \\ ${ }^{3}$ Объединенный институт высоких температур РАН, \\ 125412 Москва, Россия \\ ${ }^{4}$ Национальный исследовательский ядерный университет „МИФИ“, \\ 115409 Москва, Россия \\ e-mail: dubinov-ae@yandex.ru
}

Поступило в Редакцию 21 октября 2019 г.

В окончательной редакции 21 октября 2019г.

Принято к публикации 24 декабря 2019 г.

Путем РiC-моделирования исследована динамика процесса формирования виртуального катода и сжатого состояния пучка (ССП) в устройстве с допредельным релятивистским пучком электронов при повторном вводе электронного пучка в трубу дрейфа. Были определены физические характеристики ССП: геометрия, концентрация и температура электронов.

Ключевые слова: электронный пучок, виртуальный катод, сжатое состояние пучка, РіС-моделирования, трубу дрейфа.

DOI: $10.21883 / J T F .2020 .06 .49296 .346-19$

\section{Введение}

Сжатое состояние электронного пучка (ССП) было обнаружено при particle-in-cell-моделировании (PiC-моделирование) процессов в магнито-изолированных виркаторах [1]. Оно представляет собой плотное горячее заряженное электронное облако большой длины, сформированное в дрейфовом пространстве. Впоследствии ССП интенсивно исследовалось как экспериментально, так и помощью РіС-моделирования применительно к мощным СВЧ-генераторам виркаторного [2-4], магнетронного [5] и плазменно-пучкового типа [6], к генераторам сверхкоротких импульсов тока $[7,8]$, к коллективным ускорителям ионов [9], к источникам многозарядных ионов на базе электронных ловушек [10-12], к газоразрядным устройствам с пучково-плазменным разрядом [13] и др. Отметим, что ССП разные авторы называют иногда поразному: электронная струна [10], распределенный или протяженный (lengthy) виртуальный катод (ВК) [5,13], медленное состояние пучка (в противоположность быстрому двухпотоковому состоянию) [14].

Обычно ССП возникает из одного или нескольких ВК, а затем распространяется вдоль всего двухпотокового состояния пучка. Следовательно, для возникновения ССП необходимо, чтобы ток пучка превышал значение предельного вакуумного тока для данного дрейфового пространства.
Если заставить электронный пучок повторно или многократно проходить через одно и то же дрейфовое пространство, то можно вдвое или многократно снизить значение тока пучка для формирования ВК и ССП, так как повторно вошедшие в дрейфовом пространстве электроны увеличивают пространственный заряд и способствуют формированию ВК. Так, рождаются ВК и ССП, например, в электронных ловушках [10-12], когда пространственный заряд накапливается при многократном прохождении пучка через дрейфовое пространство с существенно меньшим предельного значения током.

В настоящей работе проведено РіС-моделирование процесса формирования ВК и ССП в длинном эквипотенциальном дрейфовом пространстве цилиндрической формы (трубе дрейфа) при повторном вводе пучка в эту трубу в случае, когда ток пучка меньше значения предельного вакуумного тока. Были определены динамические особенности этого процесса и характеристики итогового ССП.

\section{Геометрия задачи}

Существует несколько способов осуществления повторного или многократного прохождения электронным пучком дрейфового пространства. Например, можно отразить электронный пучок на выходе с помощью магнитной пробки. Такой способ исследовался в [2], 


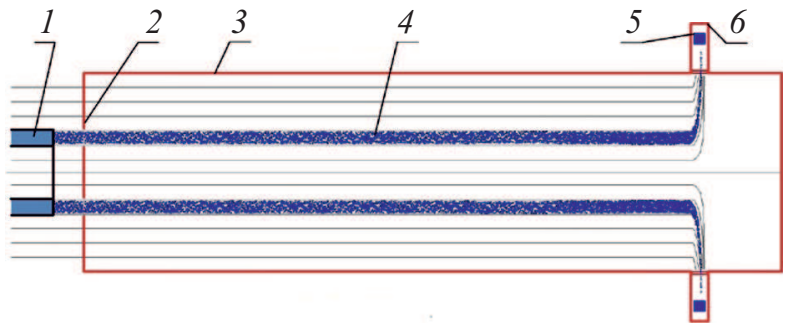

Рис. 1. Схема моделируемого устройства: 1 - катод, 2 - анодная диафрагма, 3 - труба дрейфа, 4 - электронный пучок, 5 - отражающий электрод, 6 - коллектор.

однако возможность формирования ВК и ССП в режиме допредельного тока в указанной работе не рассматривалась.

В настоящей работе предложена иная схема, в которой электронный пучок, вышедший из дрейфового пространства, разворачивается и направляется назад с помощью неоднородного магнитного поля и специального отражающего электрода, который установлен в электронном коллекторе.

Схема моделируемого устройства показана на рис. 1 . Оно содержит катод в виде полого цилиндра, замкнутую с торцов цилиндрическую трубу дрейфа, на торцах которой установлены анодная диафрагма (на рис. 1 слева), а также электронный коллектор, отделенный от трубы дрейфа прозрачной для электронов диафрагмой. Внутри коллектора находится отражающий электрод, на который подается электростатический потенциал, способный отражать электроны и направлять их внутрь трубы дрейфа. Отметим, что коллектор установлен заподлицо внутренней поверхности трубы дрейфа с тем, чтобы форма трубы дрейфа была строго цилиндрической.

Устройство находится в сильном аксиальном магнитном поле, которое в большей части однородно, но вблизи коллектора спадает до нуля. В спадающем магнитном поле линии магнитного поля направлены почти радиально, что заставляет электроны двигаться в этом месте радиально и заходить в полость коллектора. Линии поля показаны на рис. 1 тонкими кривыми.

Особое внимание уделим диафрагме на входе электронного пучка из катод-анодного промежутка в трубу дрейфа. Будем считать, что ее конструкция сделана наподобие диафрагмы редитрона [15], т. е. диафрагма свободно пропускает электроны из катод-анодного промежутка в область трубы дрейфа и полностью поглощает электроны, падающие на нее из трубы дрейфа, не пропуская их в диодную область. Такое предположение позволяет не учитывать возмущения электронного потока в диодной области электронами, вернувшимися из трубы дрейфа. Тогда при моделировании можно считать, что в трубу дрейфа поступает невозмущенный электронный пучок.

Моделирование устройства осуществлялось с помощью кода „КАРАТ“ [16], который представляет собой электромагнитный код на базе $\mathrm{PiC}$-метода. Он предназначен для решения нестационарных электродинамических задач, имеющих сложную геометрию и включающих динамику, в общем случае, релятивистских частиц (электронов, ионов, нейтралов).

Было смоделировано несколько подобных устройств с различными параметрами. Ниже в качестве примера представлены результаты моделирования одного из них, имеющего трубу дрейфа радиусом $R=10 \mathrm{~cm}$ и длиной $L=4 \mathrm{~m}$. Коллектор устанавливался на координатной отметке $z=360 \mathrm{~cm}$. Считалось, что в нулевой момент времени из диодного промежутка в трубу дрейфа инжектируется трубчатый моноэнергетический электронный пучок с постоянным по времени током $I_{b}=7 \mathrm{kA}$, внешним радиусом $r_{b}=4 \mathrm{~cm}$ и толщиной трубки тока $\Delta_{b}=1 \mathrm{~cm}$. Энергия электронов в пучке составляет $1 \mathrm{MeV}$ (лоренц-фактор $\gamma \cong 2.96$ ). Укажем, что для этих параметров трубы дрейфа и электронного пучка значение предельного вакуумного тока примерно $8.9 \mathrm{kA}$.

\section{Результаты моделирования динамики формирования ВК и ССП}

Моделирование устройства, что в пучке после его повторного вхождения в трубу дрейфа действительно возникает ВК, а затем в пучке развивается ССП. Сложную нелинейную динамику возникновения ВК и ССП удобно отслеживать по эволюции его фазового портрета. На рис. 2 показана эта эволюция с шагом $10 \mathrm{~ns}$ на протяжении 30-180 ns, т.е. на промежутке времени, пока изменения фазового портрета значительны.

Прокомментируем основные фазы эволюции фазового портрета. К $30 \mathrm{~ns}$ отраженный пучок почти полностью повторно прошел трубу дрейфа, но ВК пока еще нет, хотя есть проседания энергии электронов вблизи левого края трубы. Далее удвоенного пространственного заряда становится достаточно, чтобы к $40 \mathrm{~ns}$ в левой части трубы дрейфа сформировался ВК. Далее в течение с 50 по $80 \mathrm{~ns}$ ВК расширяется в продольном направлении, формируя ССП, которое представляет собой плотную электронную плазму. После $80 \mathrm{~ns}$ ССП начинает укорачиваться и к $120 \mathrm{~ns}$ снова возвращается по форме к ВК.

В это время к $100 \mathrm{~ns}$ с правой стороны трубы дрейфа возникает еще один ВК, который прорастает в форме 2-го участка с ССП, пока к 170 ns не заполнит всю трубу дрейфа. В дальнейшем эволюция фазового портрета прекращается.

Отметим что на фазовых портретах заметны „усы“ фазовые изображения бегущих пространственнопериодических банчей релятивистских электронов. Наличия в системе нескольких ВК и цепочки бегущих банчей указывают на то, что моделируемое устройство с допредельным пучком электронов может являться генератором мощного СВЧ-излучения. 

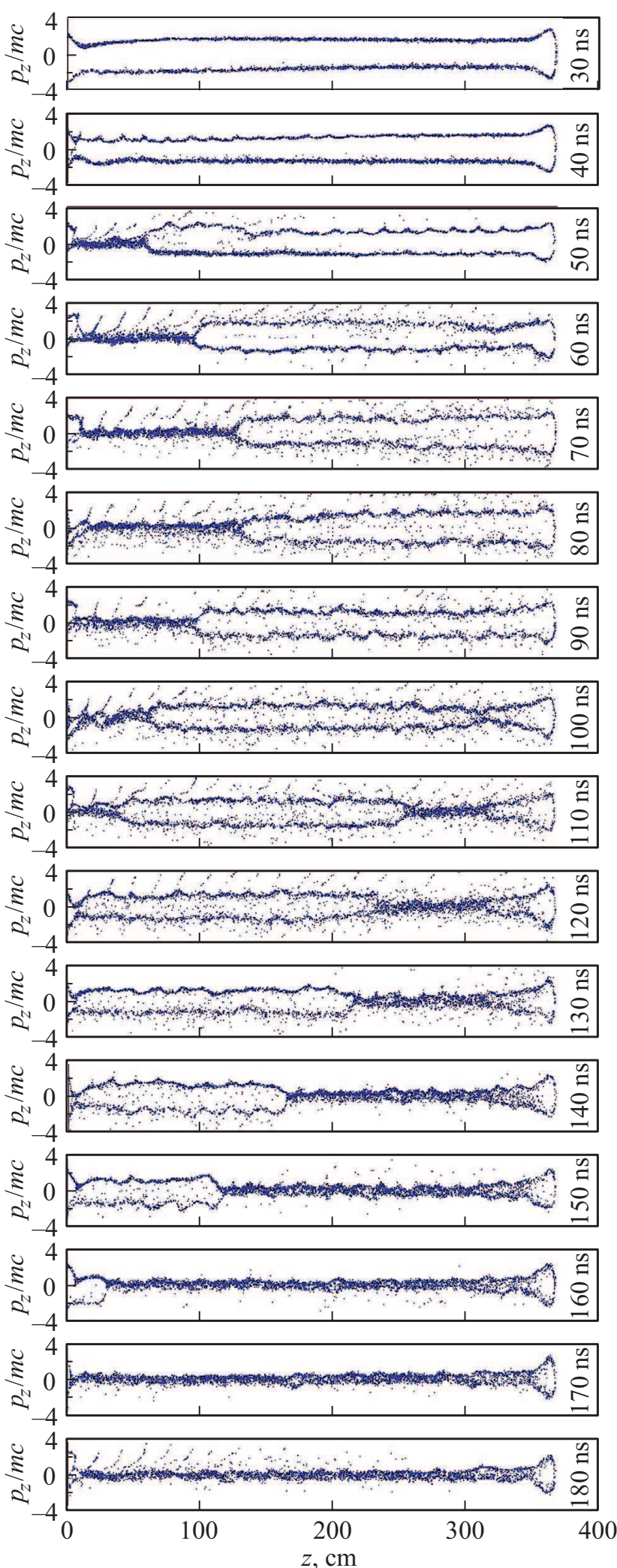

Рис. 2. Эволюция фазового портрета пучка с 30 до $180 \mathrm{~ns}$ с шагом $10 \mathrm{~ns}$ (по осям: $z$ - аксиальная координата электронов, $p_{z} / m c$ - аксиальная компонента импульса электронов, нормированная на $m c$, где $m-$ масса электрона, $c$ - скорость света).
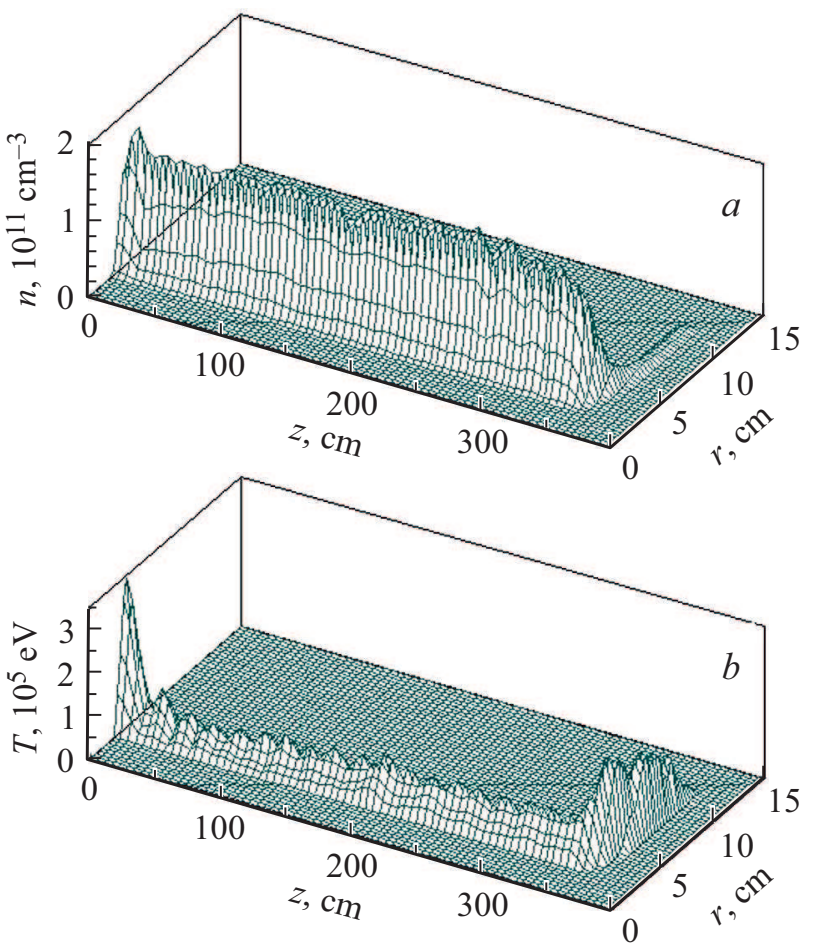

Рис. 3. Пространственные распределения физических характеристик ССП в момент времени $180 \mathrm{~ns}: a-$ концентрации электронов, $b-$ температуры электронов.

\section{Физические характеристики ССП}

В результате моделирования были определены физические характеристики ССП, которое представляет плотную горячую заряженную электронную плазму в форме тонкостенного цилиндра длиной $l \cong 350 \mathrm{~cm}$, внешним радиусом $r_{b}=4 \mathrm{~cm}$ и толщиной стенки $\Delta_{b}=1 \mathrm{~cm}$.

Концентрация электронов в нем оценена, как $n \cong 1.7 \cdot 10^{12} \mathrm{~cm}^{-3}$, а температура близка $50 \mathrm{keV}$. Профили концентрации и температуры электронов в ССП представлены на рис. 3

\section{Заключение}

Таким образом, в работе путем РiC-моделирования исследована динамика процесса формирования ВК и ССП в устройстве с допредельным релятивистским пучком электронов при повторном вводе электронного пучка в пространство дрейфа. Были определены физические характеристики ССП: геометрия, концентрация и температура электронов. Подобные устройства могут найти применение в плазменно-пучковой и вакуумной сильноточной СВЧ-электронике.

\section{Конфликт интересов}

Авторы заявляют, что у них нет конфликта интересов. 


\section{Список литературы}

[1] Ignatov A.M., Tarakanov V.P. // Phys. Plasmas. 1994. Vol. 1. N 3. P. 741

[2] Дубинов A.E. // РЭ. 2000. Т. 45. № 7. С. 875.

[3] Егоров Е.Н., Короновский А.А., Куркин С.А., Храмов А.Е. // Физ. плазмы. 2013. Т. 39. № 11. С. 1033.

[4] Dubinov A.E., Tarakanov V.P. // Laser Part. Beams. 2017. Vol. 35. N 2. P. 362.

[5] Fuks M.I., Prasad S., Schamiloglu E. // IEEE Trans. Plasma Sci. 2016. Vol. 44. N 8. P. 1298

[6] Dubinov A.E., Petrik A.G., Kurkin S.A., Frolov N.S., Koronovskii A.A., Hramov A.E. // Phys. Plasmas. 2016. Vol. 23. N 4. P. 042105.

[7] Dubinov A.E., Saikov S.K., Tarakanov V.P. // Phys. Wave Phenom. 2017. Vol. 25. № 3. P. 238.

[8] Leopold J.G., Bliokh Y.P., Siman-Tov M., Krasik Ya.E. // Phys. Plasmas. 2019. Vol. 26. N 9. P. 093107.

[9] Дубинов А.Е., Селемир В.Д., Тараканов В.П. // Письма в ЖТФ. 2002. Т. 28. Вып. 4. С. 71.

[10] Донеи, Е.Д., Донеи, Е.Е., Сыресин Е.М., Дубинов А.Е., Макаров И.В., Садовой С.А., Сайков С.К., Тараканов В.П. // Физ. плазмы. 2009. Т. 35. № 1. С. 61.

[11] Донеи, Е.Д., Донеи, Е.Е., Сыресин Е.М., Дубинов А.Е., Макаров И.В., Садовой С.А., Сайков С.К., Тараканов В.П. // ЖТФ. 2011. Т. 81. Вып. 5. С. 103-110.

[12] Дубинов А.Е., Макаров И.В., Садовой С.А., Сайков С.К., Тараканов В.П. // Письма в ЖТФ. 2011. Т. 37. Вып. 5. C. 81.

[13] Барабанов В.Н., Дубинов А.Е., Лойко М.В., Сайков С.К., Селемир В.Д., Тараканов В.П. // Физ. плазмы. 2012. Т. 38. № 2. C. 189.

[14] Беломытиев С.Я., Гришков А.А., Кицанов С.А., Коровин С.Д., Полевин С.Д., Рыжсов В.В., Ячный А.П. // Письма в ЖТФ. 2005. Т. 31. Вып. 22. С. 74.

[15] Davis H.A., Bartsch R.R., Kwan T.J.T., Sherwood E.G., Stringfield R.M. // IEEE Trans. Plasma Sci. 1988. Vol. 16. N 2. P. 192.

[16] Tarakanov V.P. User's Manual for Code KARAT. Berkley Research Associates, Springfield, VA: Berkley Research Associates, 1992. 